\title{
Patients with breast cancer may be at higher risk of colorectal neoplasia
}

\author{
Hamzah Abu-Sbeiha, Faisal S. Alib, Phillip S. Gea, Carlos H. Barcenas', Phillip Lumª, Wei Qiao d, \\ Robert S. Bresaliera, Manoop S. Bhutani ${ }^{a}$, Gottumukkala S. Rajua, Yinghong Wang ${ }^{\text {a }}$ \\ The University of Texas MD Anderson Cancer Center, Houston, Texas; Presence Saint Joseph Hospital, Chicago, IL, \\ USA
}

\section{Abstract}

Department of ${ }^{\mathrm{a}}$ Gastroenterology, Hepatology, and Nutrition, The University of Texas MD Anderson Cancer Center, Houston, Texas (Hamzah Abu-Sbeih, Phillip S. Ge, Phillip Lum, Robert S. Bresalier, Manoop S. Bhutani, Gottumukkala S. Raju, Yinghong Wang); ' Internal Medicine, Presence Saint Joseph Hospital, Chicago, IL (Faisal S. Ali); 'Breast Medical Oncology, The University of Texas MD Anderson Cancer Center, Houston, Texas (Carlos H. Barcenas); ${ }^{\mathrm{d} B i o s t a t i s t i c s, ~}$ The University of Texas MD Anderson Cancer Center, Houston, Texas (Wei Qiao), USA

\section{Conflict of Interest: None}

Correspondence to: Yinghong Wang, MD, Department of Gastroenterology, Hepatology and Nutrition, The University of Texas MD Anderson Cancer Center, 1515 Holcombe Blvd., Unit 1466, Houston, TX 77030, USA, e-mail: ywang59@mdanderson.org

Received 14 March 2019; accepted 17 April 2019; published online 20 May 2019

DOI: https://doi.org/10.20524/aog.2019.0387

\section{Introduction}

With advances in cancer therapeutics and the development of early screening and cancer detection, as well as a multidisciplinary approach to therapy, the 5-year survival rates of patients with localized breast cancer and breast cancer spread to regional lymph nodes have improved to $98.7 \%$ and $85.3 \%$, respectively; this translates into 2.8 million breast cancer survivors [1]. With such a significant proportion of survivors, it is imperative to ensure that the long-term wellbeing of these patients is ensured, particularly by active surveillance for the long-term consequences of breast cancer and the cancer therapies these patients were exposed to. One of the most concerning potential threats to the long-term wellbeing of cancer survivors is the occurrence of a second de novo malignancy. 
Colorectal cancer (CRC) is among the most common cancers affecting the American population. With more than 140,250 Americans diagnosed annually and a 5-year survival of $64.5 \%$, CRC remains a challenge for clinical oncologists and imposes a significant burden on the patients' well-being [2]. There has been speculation regarding the incidence of nonbreast malignancies, and particularly CRC, among patients with a history of breast cancer, though the literature on this topic has been conflicting [3-5]. A positive correlation between breast cancer and CRC was found after pooling the findings of 5 cross-sectional and case-control studies, with a pooled relative risk of 1.74 (95\% confidence interval [CI] 1.27-2.21) [6]. On the other hand, more recent case-control studies have questioned the validity of a presumed higher risk of CRC among breast cancer survivors [7-9].

The greater risk of CRC in breast cancer survivors has been attributed to conditions such as obesity, hormonal influences, and the potential genetic commonality in the origin of breast and colon oncogenesis, as is seen among patients with familial cancer syndromes such as hereditary nonpolyposis CRC [10-12]. However, none of these factors addresses the incidence of adenomatous polyps and their progression to CRC among patients with a history of breast cancer. The adenoma detection rate (ADR) in our institution for patients $50-70$ years old (38\%) is higher than the reported ADR in the general population (25-35\%). Preliminary evidence in the form of case-control studies reveals conflicting results concerning the risk of developing adenomatous polyps in patients with a history of breast cancer [13-18]. Additionally, the current literature consists of studies with small sample sizes, and the body of evidence addressing this issue is limited.

The aim of this study was to determine the incidence of adenomatous polyps and CRC among patients with a history of breast cancer in a tertiary cancer center, which receives a high volume of breast cancer patients.

\section{Patients and methods}

This was a retrospective study of patients with a diagnosis of breast cancer who had undergone a colonoscopy after their breast cancer diagnosis between 2000 and 2017. This study was approved by the Institutional Review Board at The University of Texas MD Anderson Cancer Center. Eligible patients were identified from our institutional tumor registry and endoscopy unit database. All patients had confirmed malignancy of the breast by histopathology. Patients with benign breast lesions or a personal history of CRC prior to breast cancer diagnosis were excluded. Our control cohort included all patients without a personal history of any type of cancer who underwent colonoscopic screening between 2008 and 2017.

Natural language processing and electronic medical chart review were conducted to extract information pertaining to demographics and medical and oncologic history. Demographics included age, sex, race/ethnicity, and body mass index (BMI) at the time of colonoscopy. Medical and oncologic history consisted of history of smoking, a first- degree relative with a history of CRC, breast cancer treatment modality, site of breast cancer and histologic type of breast cancer. The hormonal status of breast cancer (i.e., estrogen and progesterone positivity) was extracted from the breast cancer departmental database.

\section{Colonoscopy data}

The index colonoscopy performed after breast cancer diagnosis was recorded. Patients with poor preparation for the colonoscopy were excluded. The time from breast cancer diagnosis to first colonoscopy was calculated. Data regarding the colonoscopic assessment included the size of the polyp, the site of the polyp, the morphology of the polyp, and the method of polyp removal. The size of the polyp was divided into small $(\leq 10 \mathrm{~mm})$ or large $(>10 \mathrm{~mm})$. The site of the polyp was described as left colon if it was located distal to the splenic flexure and as right colon if it was located proximal to the splenic flexure. Sessile, pedunculated, flat, and diminutive or indeterminate were the morphological categories of the polyps.

Histologic evaluation was performed for all colonic lesions. The histologic subgroups of the colonic adenoma consisted of tubular, tubulovillous, villous, or serrated. The presence of high-grade dysplasia or advanced adenocarcinoma features in the colonic tissue was reported. Patients with inadequate histologic specimens were excluded. ADR was defined as the percentage of procedures where colonic adenomas were detected divided by the total number of procedures.

For patients in whom adenoma was detected during index colonoscopy, data regarding subsequent surveillance colonoscopies were also recorded and the ADR for all subsequent colonoscopies was documented. For these patients, the adenoma recurrence-free time was defined as the period from the index colonoscopy to the first subsequent colonoscopy with adenoma or the last subsequent colonoscopy without adenoma, whichever was earlier.

\section{Statistical analysis}

Descriptive statistical measures using means and standard deviations or medians with interquartile ranges for continuous variables were conducted. Categorical variables were summarized using frequencies and percentages. Fisher's exact and $\chi^{2}$ tests were used to compare categorical variables. Wilcoxon rank sum and Kruskal-Wallis tests were used to compare continuous variables. To assess for factors associated with adenoma and adenoma recurrence on the repeat colonoscopy, univariate and multivariate logistic regression analyses were conducted. To assess predictors of early adenoma recurrence, we performed Cox regression model. Statistical tests were two-sided. P-values of 0.05 or less were considered statistically significant. Statistical analysis was carried out using SAS (version 9.4; SAS Institute, Cary, NC, USA) and SPSS (version 24.0; IBM Corporation, Armonk, NY, USA) software. 


\section{Results}

\section{Patient characteristics}

Of the 62,820 patients who had a breast cancer diagnosis during the study period, 3304 met the inclusion criteria. Most were female $(99.6 \%)$, the mean age was 59 years (standard deviation, 10 years) and the majority were white (68.2\%). A first-degree relative with a history of CRC was reported in $43.0 \%$ of patients and a history of smoking in $36.7 \%$ (Table 1 ). The clinical characteristics of patients in the control group $(n=3295)$ compared with those of patients with breast cancer are listed in Supplemental Table 1.

\section{Breast cancer history}

Most patients had their breast lesion removed surgically without adjuvant therapy (56\%). Of the patients who did receive adjuvant therapy, $10 \%$ received cytotoxic chemotherapy only and $38 \%$ received hormonal therapy in addition to cytotoxic therapy. Ductal carcinoma was the most common histologic type (65\%), followed by lobular carcinoma (12\%).

\section{Colon polyps}

Colon adenomas were detected in 1803 patients (55\%) with breast cancer (Supplemental Table 2). Of these, 575 patients had more than one adenoma. Patients with colonic adenomas were older $(\mathrm{P}=0.001)$, had higher $\mathrm{BMI}(\mathrm{P}=0.004)$, and reported a history of $\mathrm{CRC}$ in a first-degree relative more frequently $(\mathrm{P}<0.001)$ compared to patients without adenomas (Table 1$)$. The median time from breast cancer diagnosis to first colonoscopy was 3 years (interquartile range, 1-6). The ADR for the control group was $29 \%$.

Features of the colonic adenomatous polyps $(n=2720$ polyps) found in breast cancer patients are listed in Table 2.

Table 1 Characteristics of breast cancer patients with and without adenomatous polyps detected by colonoscopy (N=3304)

\begin{tabular}{|c|c|c|c|}
\hline Characteristic & Polyps, n (\%), (N=1803) & No polyps, $\mathrm{n}(\%),(\mathrm{N}=1501)$ & $\mathrm{P}$ \\
\hline Age, years, mean (SD) & $60.6(9.1)$ & $58.1(9.9)$ & $<0.001$ \\
\hline Female & $1796(99.6)$ & $1494(99.5)$ & 0.792 \\
\hline Race & & & 0.716 \\
\hline White & $1235(68.5)$ & $1018(67.8)$ & \\
\hline Black & $255(14.1)$ & $223(14.9)$ & \\
\hline Hispanic & $204(11.3)$ & $158(10.5)$ & \\
\hline Asian & $90(5.0)$ & $80(5.3)$ & \\
\hline Other & $19(1.1)$ & $22(1.5)$ & \\
\hline BMI, kg/m², mean (SD) & $30(7)$ & $29(7)$ & 0.004 \\
\hline $\mathrm{BMI}>30 \mathrm{~kg} / \mathrm{m}^{2}$ & $710(43.4)$ & $523(40.0)$ & 0.065 \\
\hline Family history of colorectal cancer & $827(45.9)$ & $595(39.6)$ & $<0.001$ \\
\hline Smoking & $668(37.0)$ & $544(36.2)$ & 0.638 \\
\hline Breast cancer treatment & & & 0.394 \\
\hline Surgery only & $982(54.5)$ & $853(56.8)$ & \\
\hline Chemotherapy & $115(6.4)$ & $90(6.0)$ & \\
\hline Endocrine therapy & $706(39.2)$ & $558(37.2)$ & \\
\hline Type of breast cancer & & & 0.284 \\
\hline Ductal & $1166(64.7)$ & $983(65.5)$ & \\
\hline Lobular & $220(12.2)$ & $166(11.1)$ & \\
\hline Mixed & $192(10.6)$ & $137(9.1)$ & \\
\hline Inflammatory & $21(1.2)$ & $21(1.4)$ & \\
\hline Other & $204(11.3)$ & $194(12.9)$ & \\
\hline \multicolumn{4}{|c|}{ Breast cancer hormonal status $(n=2617)$} \\
\hline Estrogen positive & $1149(79.8)$ & $929(78.9)$ & 0.593 \\
\hline Progesterone positive & $957(66.9)$ & $760(65.2)$ & 0.382 \\
\hline Either or both & $1171(81.3)$ & $951(80.8)$ & 0.763 \\
\hline
\end{tabular}


Most polyps were located in the right colon (64\%); of these, detected in $8 \%$ and villous features in $1 \%$ of polyps. Serrated adenoma was detected in 176 polyps (7\%). High-grade dysplasia was evident in $28 \%$ of polyps. Invasive adenocarcinoma was detected in 172 patients (5\%).

\section{ADR by age and time from breast cancer diagnosis to colonoscopy}

The ADR was $21 \%$ in the 63 patients younger than 40 years, $39 \%$ in the 314 patients between 40 and 50 years, $54 \%$ in the 1420 patients between 51 and 60 years, and $60 \%$ in the 1507 patients older than 60 years. ADRs of patients stratified by age and time from breast cancer diagnosis to colonoscopy are shown in Fig. 1. In patients 50 years or younger, the ADR was the highest if the colonoscopy occurred more than 10 years after breast cancer diagnosis. Among the same age group, the ADR was only $26 \%$ in patients who did not have a family history of CRC or a BMI higher than $30 \mathrm{~kg} / \mathrm{m}^{2}$ (Supplemental Fig. 1).

\section{Factors associated with adenoma} both patients with a history of breast cancer and the control group, older age, higher BMI, family history of colorectal cancer in a first-degree relative, and a personal history of breast cancer were associated with a higher risk of colon adenoma (Table 3).

\section{Repeated colonoscopy evaluation}

A subsequent colonoscopy for any indication was performed in 831 patients in whom adenomas had been detected on the

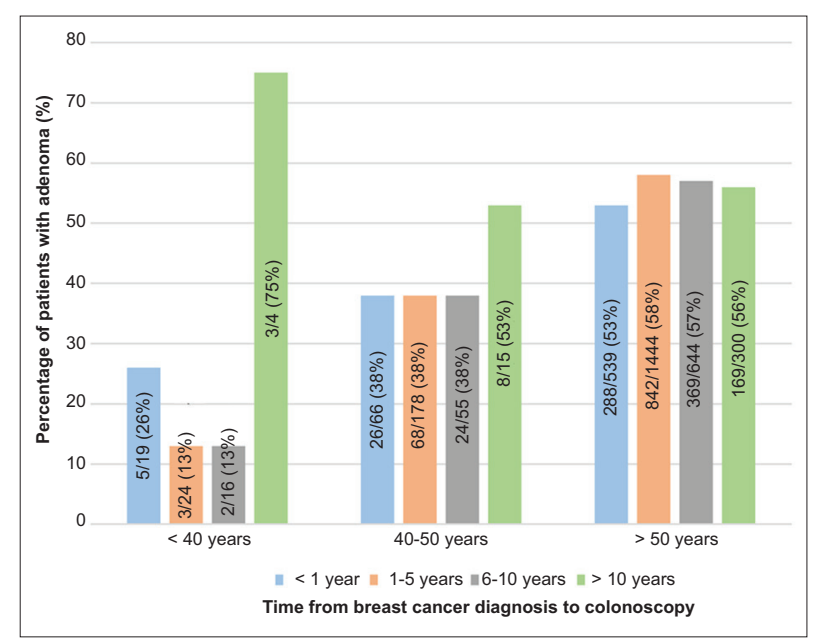

Figure 1 Adenoma detection rate among patients with breast cancer stratified by age and time from breast cancer diagnosis to colonoscopy 135 (5\%) were larger than $10 \mathrm{~mm}$. Tubulovillous features were

On multivariate logistic regression analysis that included

initial colonoscopy. The ADR for the repeated colonoscopy was higher for patients whose second colonoscopy occurred more than 5 years after the first than for patients with shorter intervals (Table 4). The ADR for the repeated colonoscopy was higher $(54 \%)$ for patients whose polyps had high-risk features for adenoma recurrence (i.e. more than 2 polyps,

Table 2 Polyp characteristics among patients with breast cancer

\begin{tabular}{lc}
\hline Characteristic & Polyps, $\mathrm{n}(\%)(\mathrm{N}=2720)$ \\
\hline Site & $1737(63.9)$ \\
Right colon & $983(36.1)$ \\
Left colon & \\
Size & $135(5.0)$ \\
$>10$ mm & $2585(95.0)$ \\
$\leq 10$ mm & \\
Morphology & $203(7.5)$ \\
Pedunculated & $1088(40.0)$ \\
Sessile & $238(8.8)$ \\
Flat & $1191(43.8)$ \\
Diminutive & \\
Endoscopic removal & $1799(66.1)$ \\
Biopsy/forceps & $362(13.3)$ \\
Hot snare & $387(14.2)$ \\
Cold snare & $138(5.1)$ \\
En bloc EMR & $34(1.3)$ \\
Piecemeal EMR & \\
Histologic features & $172(6.3)$ \\
Tubular & $2305(85.7)$ \\
Tubulovillous & $225(8.3)$ \\
Villous & $14(0.5)$ \\
Serrated & $176(6.5)$ \\
High grade dysplasia & \\
Cancer & \\
\hline EMR, endoscopic mucosal resection & \\
\hline & \\
\hline & \\
\hline
\end{tabular}

Table 3 Multivariate logistic regression: risk factors of adenoma (all patients, $\mathrm{n}=6599$ )

\begin{tabular}{lll}
\hline \multirow{2}{*}{ Characteristic } & \multicolumn{2}{c}{ Adenoma } \\
\cline { 2 - 3 } & OR $(95 \% \mathrm{CI})$ & $\mathrm{P}$ \\
\hline Age & $1.04(1.03-1.05)$ & $<0.001$ \\
Male sex & $0.60(0.19-1.93)$ & 0.393 \\
BMI & $1.01(1.00-1.03)$ & 0.022 \\
Family history of colorectal cancer & $1.30(1.10-1.53)$ & 0.002 \\
\hline Smoking & $1.08(0.91-1.28)$ & 0.363 \\
Breast cancer & $2.97(2.68-3.28)$ & $<0.001$ \\
\hline Positive hormonal status & $0.94(0.76-1.18)$ & 0.608 \\
\hline BMI body mass index. OR, odds ratio $C I$, confidence interval
\end{tabular}


polyps larger than $10 \mathrm{~mm}$, and histological confirmation of high-grade dysplasia or tubulovillous or villous features) than for patients without high-risk features $(41 \%$; $\mathrm{P}<0.001)$. Furthermore, the median adenoma recurrence-free time was 3 years (interquartile range 2-5), and it was shorter for patients whose polyps had high-risk features (3 years; interquartile range 2-4) than for patients whose polyps did not have such features (4 years; interquartile range $2-5$; $\mathrm{P}<0.001$ ).

\section{Factors associated with adenoma recurrence}

A first-degree relative with a history of CRC, a personal history of breast cancer, more than one polyp detected on the initial colonoscopy, and a polyp larger than $10 \mathrm{~mm}$ on the initial colonoscopy were factors associated with a high risk of adenoma recurrence (Table 5).

\section{Factors associated with earlier adenoma recurrence}

Old age, more than two polyps detected on initial colonoscopy and large (>10 $\mathrm{mm}$ ) polyps on initial colonoscopy were associated with early adenoma recurrence (Table 6).

\section{Discussion}

This single-center retrospective study from a tertiary cancer center study assessed the incidence of adenomatous polyps in patients who had a history of breast cancer. Adenomatous polyps were detected in $55 \%$ of the study population, higher than the median ADR at our institution (38\%). The ADR in patients younger than 40 years and between 40 and 50 years was $21 \%$ and $39 \%$, respectively. Among patients younger than 50 years, the ADR was highest $(>50 \%)$ among patients whose colonoscopy occurred 10 years after the breast cancer diagnosis. Our multivariate logistic regression analysis comparing patients with breast cancer to a control cohort also identified breast cancer as an independent risk factor for colon adenomas.

Our findings contradict the previously reported ADR among patients with a history of breast cancer. A meta-analysis of 6 case-control studies that reported ADR in patients with breast cancer revealed 67 and 47 incidences of polyp detection among cases and controls, respectively, translating into a pooled odds ratio of $1.5 \%$ (95\%CI $0.97-2.2 ; \mathrm{P}=0.07)$, with no heterogeneity $\left(I^{2}=1 \%\right)$ [13-18]. It should be noted that this analysis only included polyps larger than $1 \mathrm{~cm}$, an approach that would fail to account for smaller polyps, which according to our study constitute the majority of adenomatous polyps in breast cancer patients. Additionally, the ADRs in these case-control studies could have been influenced by a plethora of factors, including the recognized facets of a high-quality screening colonoscopy as well as the institutional ADR, not explicitly reported in these 6 studies. At our institution, the
Table 4 Adenoma detection rate by interval between first and second adenoma detection among patients with breast cancer

\begin{tabular}{lcc}
\hline $\begin{array}{l}\text { Time from first to } \\
\text { second colonoscopy }\end{array}$ & No. of patients & $\begin{array}{c}\text { Repeat } \\
\text { colonoscopy ADR }\end{array}$ \\
\hline$<3$ years & 346 & $138(39.9 \%)$ \\
$3-5$ years & 279 & $138(49.5 \%)$ \\
$>5$ years & 206 & $109(52.9 \%)$ \\
\hline ADR, adenoma detection rate & &
\end{tabular}

Table 5 Logistic regression: risk factors for adenoma recurrence

\begin{tabular}{lcc}
\hline \multirow{2}{*}{ Characteristic } & \multicolumn{2}{c}{ Adenoma recurrence } \\
\cline { 2 - 3 } & OR (95\%CI) & $\mathrm{P}$ \\
\hline Age & $1.01(0.99-1.03)$ & 0.223 \\
BMI & $1.01(0.98-1.03)$ & 0.563 \\
Family history of colorectal cancer & $1.68(1.24-2.72)$ & 0.001 \\
Smoking & $1.03(0.76-1.39)$ & 0.852 \\
Number of polyps & $1.84(1.50-2.25)$ & $<0.001$ \\
High-grade dysplasia & $0.99(0.67-1.48)$ & 0.984 \\
\hline Polyp larger than 10 mm & $1.24(0.67-2.28)$ & 0.490 \\
Tubulovillous or villous features & $1.55(0.98-2.46)$ & 0.061 \\
\hline $\begin{array}{l}\text { Time from first to second } \\
\text { colonoscopy }\end{array}$ & $1.12(1.05-1.21)$ & 0.001 \\
\hline Breast cancer & $1.92(1.59-2.32)$ & $<0.001$ \\
\hline BMI, body mass index; OR, odds ratio; CI, confidence interval & \\
\hline
\end{tabular}

Table 6 Cox regression: time to adenoma recurrence

\begin{tabular}{lcc}
\hline \multirow{2}{*}{ Characteristic } & \multicolumn{2}{c}{ Time to recurrence } \\
\cline { 2 - 3 } & OR $(95 \% \mathrm{CI})$ & $\mathrm{P}$ \\
\hline Age & $1.01(1.00-1.03)$ & 0.044 \\
$\begin{array}{l}\text { Family history of colorectal } \\
\text { cancer }\end{array}$ & $1.17(0.95-1.44)$ & 0.137 \\
Adenoma initially & $1.30(1.11-1.52)$ & 0.001 \\
High-grade dysplasia & $1.01(0.79-1.30)$ & 0.926 \\
Number of polyps & $1.40(1.29-1.51)$ & $<0.001$ \\
Polyp larger than 10 mm & $1.73(1.19-2.53)$ & 0.004 \\
Tubulovillous or villous features & $1.19(0.89-1.59)$ & 0.250 \\
Breast cancer & $1.03(0.89-1.19)$ & 0.698 \\
\hline
\end{tabular}

OR, odds ratio; CI, confidence interval

endoscopist turnover rate is low, which ensures consistency in the quality of endoscopy.

A significant proportion of the patients with adenomatous polyps had a first-degree relative with a history of CRC, were older, and had a higher BMI compared to patients without polyps. A higher BMI could act as a driver for their breast cancer and the development of adenomatous polyps. This finding conflicts with the hormonal aspect of colonic adenoma. Nonetheless, the hormonal status of breast cancer was not associated with a 
greater risk of colorectal neoplasia in our cohort. Additionally, a history of CRC in a first-degree relative hints at a genetic susceptibility to the development of adenomatous polyps. The potential influence from obesity and a positive family history is further supported by the fact that, for patients younger than 50 years who were not obese or did not have a family history of $\mathrm{CRC}$, the ADR was only $26 \%$. However, since the majority of the patients were not referred for genetic counseling and testing, we were unable to evaluate the presence of genetic mutations and asymptomatic familial syndromes. This is reflective of real-world practice, in which the threshold for referral to conduct genetic testing for familial cancer syndromes or genetic susceptibility to the development of adenomatous polyps is high. Even among patients with a high risk of CRC, assessed by validated criteria for hereditary cancer syndromes such as hereditary nonpolyposis $\mathrm{CRC}$, it has been reported that referral for genetic work-up and counseling is suboptimal $[19,20]$.

Most of the polyps in our study were smaller than $10 \mathrm{~mm}$, and $40 \%$ were sessile. Both of these factors have been shown to contribute to the polyp miss rate [21,22]. Additionally, a majority of the polyps were located in the right colon, which can potentially amplify the polyp miss rate if a flexible sigmoidoscopy is chosen over a colonoscopy. These factors should be taken into account in order to improve the ADR of colonoscopy, since detection of such smaller and inconspicuous polyps may require a more thorough colonic assessment, which in turn increases withdrawal time [21]. Furthermore, $6.5 \%$ of the polyps had a serrated histologic appearance. Serrated polyp detection may also require thorough colonic inspection, which further underscores the importance of a thorough colonoscopy.

Our findings also have implications for the timing of screening colonoscopy in patients with a history of breast cancer. Because the ADR for patients younger than 40 years was highest when the colonoscopy occurred more than 10 years after a breast cancer diagnosis, screening should be performed less than 10 years from the date of breast cancer diagnosis, or when the patient is 40 years old at the latest. It should be noted that, as most patients younger than 40 years without a family history of breast cancer will probably have undergone colonoscopy for gastrointestinal symptoms, this might have led to underestimation of the ADR in this patient population undergoing colonoscopy within 10 years of breast cancer diagnosis. However, for patients 40-50 years old, the ADR within 1 year of breast cancer diagnosis was $38 \%$. Hence, the appropriate timing for a recommendation of screening colonoscopy should be dictated by the patient's age at the time of breast cancer diagnosis, and a screening colonoscopy within 1 year of breast cancer diagnosis for patients older than 40 years could be deemed appropriate.

The ADR for repeat colonoscopy increased as the time from index polyp detection to repeat colonoscopy increased, ranging from $40 \%$ when performed within 3 years of the index polyp detection to $53 \%$ when performed more than 5 years from the index polyp detection. Factors associated with early adenoma recurrence should be taken into account when making recommendations regarding the optimal surveillance interval. Our analysis revealed an association between early adenoma recurrence and old age and large and multiple polyps at initial colonoscopy. A personal history of breast cancer was a risk factor for adenoma recurrence. Because the median adenoma recurrence-free time in our study was 3 years, repeat colonoscopy should be performed within 3 years of the index polyp removal.

In our study, the incidence of CRC among breast cancer survivors was $5 \%$, higher than the $1.33 \%$ rate predicted by SEER for CRC incidence among women [23]. The high rate of invasive CRC identified in our study is further grounds for suggesting early screening colonoscopy for patients with breast cancer. The authors of previous studies have advocated for early screening colonoscopy for CRC in patients with a history of breast cancer. However, the literature on this issue has been conflicting. A meta-analysis of $1,055,917$ patients with breast cancer found the pooled event rate for CRC to be $0.7 \%$ [18]. The pooled event rate in an analysis of 10 studies from the United States revealed an incidence of $1.1 \%$. It should be noted that there was significant heterogeneity among the studies included in this meta-analysis $\left(I^{2}=97 \%\right)$. As a tertiary cancer center, we had the advantage of a larger sample size of local patients, as well as national and international referrals, potentially reflecting the real-world breast cancer population.

Our study has some limitations that should be noted. The flaws of a retrospective design are inherent to our study. Additionally, subgroup analysis stratifying patients according to ethnicity was not performed; hence, it is not evident whether ethnicity plays a role in the development of adenomatous polyps in patients with breast cancer. In certain subgroups, a small sample size limited our ability to draw meaningful conclusions. As a considerable number of our cohort had colonoscopy for indications other than $\mathrm{CRC}$ screening, the ADR could have been underestimated. Lastly, although our low gastroenterologist turnover assures consistency in the quality of colonoscopy, we did not assess the impact of specific quality indicators, such as cecal intubation and withdrawal time, on the ADR.

In conclusion, patients with a history of breast cancer have a high incidence of adenomatous polyps in all age groups and a 5\% incidence of invasive CRC. Screening colonoscopy for adenoma should be considered within 5 years of breast cancer diagnosis or by the age of 40 years, whichever comes first, for patients younger than 40 years at the time of breast cancer diagnosis. For patients older than 40 years, a screening colonoscopy within 1 year of breast cancer diagnosis would be appropriate. For patients in whom adenoma is detected, a repeat colonoscopy should be performed within 3 years of the index polyp detection. For patients in whom no adenoma is detected, a surveillance interval of 5 years may suffice. Future prospective studies with adequate sample size should be performed to validate our findings regarding the risk of developing adenomatous polyps and invasive CRC among breast cancer patients compared with a well-matched control group.

\section{Acknowledgment}

Medical editing of this paper was provided by the Department of Scientific Publications at MD Anderson Cancer Center. 


\section{Summary Box}

\section{What is already known:}

- Breast cancer is among the most common malignancies

- It has been suggested that breast cancer and its treatment may be linked with colorectal neoplasia

- Studies have revealed conflicting results regarding this topic

\section{What the new findings are:}

- The rate of colon adenoma, a precursor for colorectal cancer, was higher in breast cancer survivors than in a cancer-free control cohort

- The rates of high-grade dysplasia and of colorectal cancer were also higher than in the control group

- Therefore, in breast cancer survivors, screening colonoscopy for colorectal cancer should be considered earlier than the age of 50 years, the age for colorectal cancer screening recommended by multiple societies

\section{References}

1. Female Breast Cancer - Cancer Stat Facts. Available from: https://seer. cancer.gov/statfacts/html/breast.html [Accessed 4 January 2019].

2. Colorectal Cancer - Cancer Stat Facts. Available from: https://seer. cancer.gov/statfacts/html/colorect.html [Accessed 4 January 2019].

3. Harvey EB, Brinton LA. Second cancer following cancer of the breast in Connecticut, 1935-82. Natl Cancer Inst Monogr 1985;68:99-112.

4. Schaapveld M, Visser O, Louwman MJ, et al. Risk of new primary nonbreast cancers after breast cancer treatment: a Dutch population-based study. J Clin Oncol 2008;26:1239-1246.

5. Evans HS, Lewis CM, Robinson D, Bell CM, Møller H, Hodgson SV. Incidence of multiple primary cancers in a cohort of women diagnosed with breast cancer in southeast England. $\mathrm{Br} J$ Cancer 2001;84:435-440.

6. Eisen GM, Sandler RS. Are women with breast cancer more likely to develop colorectal cancer? Critical review and meta-analysis. J Clin Gastroenterol 1994;19:57-63.
7. Srinivasan R, Yang YX, Rubin SC, Morgan MA, Lewis JD. Women with a prior diagnosis of breast cancer are not at an increased risk for subsequent colorectal cancer. Am J Gastroenterol 2005;100:2759-2764.

8. Shukla A, Shukla S, Osowo A, Mashtare T, Bhutani MS, Guha S. Risk of colorectal adenomas in women with prior breast cancer. Dig Dis Sci 2012;57:3240-3245.

9. Tang LY, Nugent Z, Demers AA, Singh H. Incidence of right-sided colorectal cancer after breast cancer: a population-based study. Am J Gastroenterol 2009;104:1213-1220.

10. Roberts ME, Jackson SA, Susswein LR, et al. MSH6 and PMS2 germ-line pathogenic variants implicated in Lynch syndrome are associated with breast cancer. Genet Med 2018;20:1167-1174.

11. Boyd J, Rhei E, Federici MG, et al. Male breast cancer in the hereditary nonpolyposis colorectal cancer syndrome. Breast Cancer Res Treat 1999;53:87-91.

12. Risinger JI, Barrett JC, Watson P, Lynch HT, Boyd J. Molecular genetic evidence of the occurrence of breast cancer as an integral tumor in patients with the hereditary nonpolyposis colorectal carcinoma syndrome. Cancer 1996;77:1836-1843.

13. Jouin $\mathrm{H}$, Baumann $\mathrm{R}$, Derlon $\mathrm{A}$, et al. Is there an increased incidence of adenomatous polyps in breast cancer patients? Cancer 1989;63:599-603.

14. Ochsenkühn T, Bayerdörffer $\mathrm{E}$, Meining $\mathrm{A}$, et al. Increased prevalence of colorectal adenomas in women with breast cancer. Digestion 2005;72:150-155.

15. Sanchez-Mete L, Venturo I, Papaldo P, Sperduti I, Stigliano V. Colorectal cancer after breast cancer: a case-control study. Cancer Epidemiol 2011;35:44-47.

16. Bremond A, Collet P, Lambert R, Martin JL. Breast cancer and polyps of the colon. A case-control study. Cancer 1984;54:2568-2570.

17. Rex DK, Sledge GW, Harper PA, et al. Colonic adenomas in asymptomatic women with a history of breast cancer. Am J Gastroenterol 1993;88:2009-2014.

18. Lai JH, Park G, Gerson LB. Association between breast cancer and the risk of colorectal cancer. Gastrointest Endosc 2017;86:429-441.

19. Nathanson JW, Zisman TL, Julian C, McCaffrey S, Rubin DT. Identification of patients at increased risk for colorectal cancer in an open access endoscopy center. J Clin Gastroenterol 2008;42:1025-1031.

20. Moole S, McGarrity TJ, Baker MJ. Screening for familial colorectal cancer risk amongst colonoscopy patients new to an open-access endoscopy center. ISRN Gastroenterol 2012;2012:152980.

21. van Rijn JC, Reitsma JB, Stoker J, Bossuyt PM, van Deventer SJ, Dekker E. Polyp miss rate determined by tandem colonoscopy: a systematic review. Am J Gastroenterol 2006;101:343-350.

22. Lee J, Park SW, Kim YS, et al. Risk factors of missed colorectal lesions after colonoscopy. Medicine (Baltimore) 2017;96:e7468.

23. Cancer Statistics Review, 1975-2015 - SEER Statistics. Available from: https://seer.cancer.gov/csr/1975_2015/[Accessed 4 January 2019]. 


\section{Supplementary Figure}

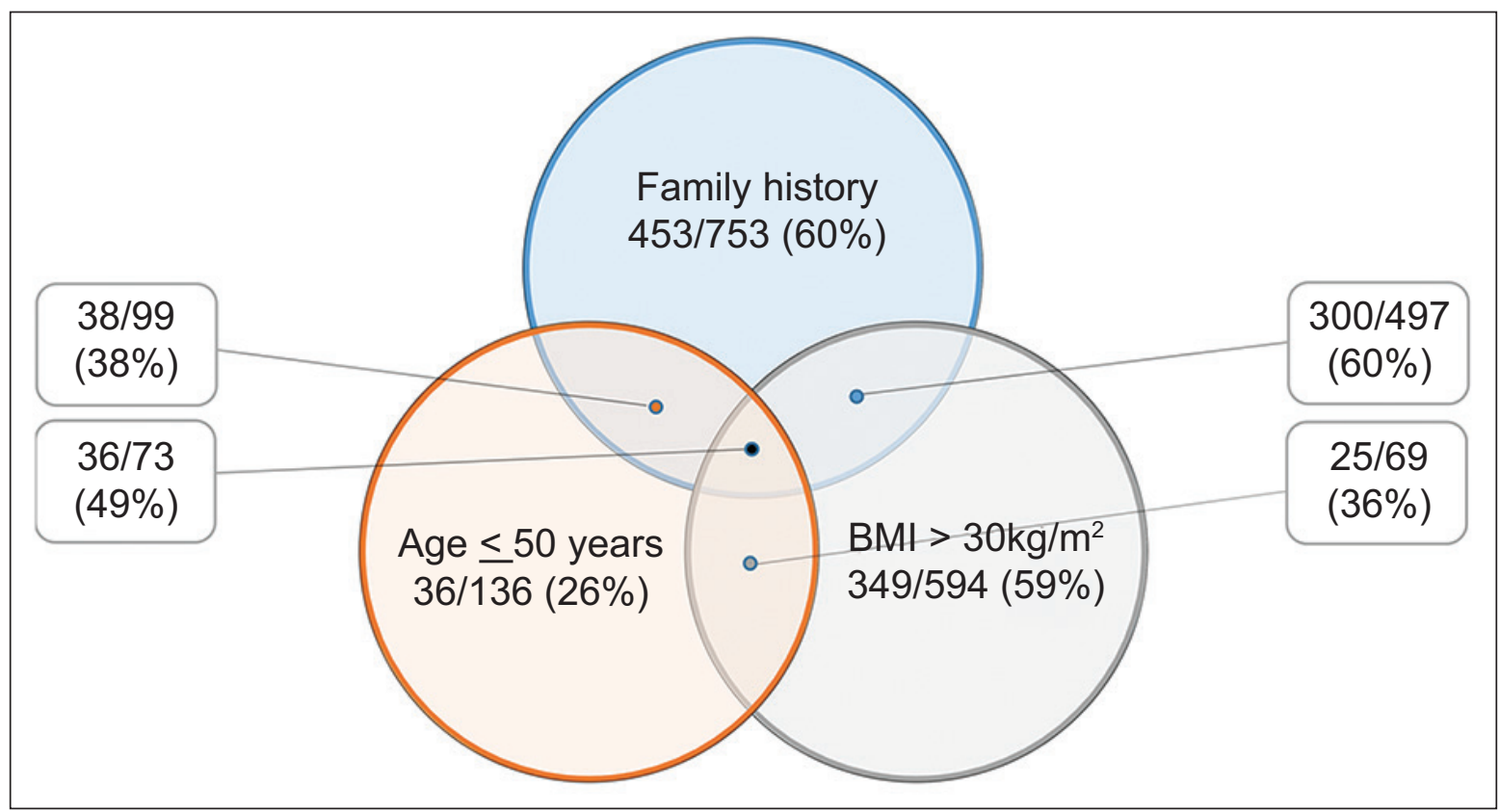

Supplementary Figure 1 Venn diagram of adenoma detection rate according to the presence of risk factors (age, family history, and body mass index [BMI]) among patients with breast cancer 